\title{
Instantaneous closed loop control of the Navier-Stokes system
}

\author{
M. Hinze \\ Technische Universität Dresden \\ Institut für Numerische Mathematik \\ Zellescher Weg 12-14 \\ D-01062 Dresden, Germany \\ email: hinze@math.tu-dresden.de
}

\begin{abstract}
Instantaneous control is applied to the control of the instationary Navier-Stokes system. This control technique is closely related to receding horizon control and allows for an interpretation as suboptimal closed loop controller, whose parameters may be adjusted so as to stabilize the nonlinear equation under consideration. Besides stability analysis for the distributed control case numerical examples for the continuous and discrete-in-time control laws are presented.
\end{abstract}

Keywords: Optimal control, Instantaneous control, Closed loop control, Navier-Stokes equations. AMS classification: 49N90, 76D05, 93B52, 93C10.

\section{Introduction}

This research is devoted to the study of instantaneous control applied to the instationary NavierStokes system. It thereby builds upon, and extends results of [14] for the Burgers equation, where also a comprehensive discussion of the method can be found.

In primal variables the Navier-Stokes system can be written in the form

$$
\left\{\begin{aligned}
y_{t}-\nu \Delta y+(y \nabla) y+\nabla p & =\mathcal{B} u & & \text { in } Q, \\
-\operatorname{div} y & =0 & & \text { in } Q, \\
y & =0 & & \text { on }(0, T) \times \partial \Omega, \\
y(0) & =\phi & & \text { in } \Omega .
\end{aligned}\right.
$$

Here $\nu:=1 /$ Re denotes the viscosity parameter, with Re denoting the Reynolds number. Furthermore, $Q:=(0, T) \times \Omega$ with $\Omega \subset \mathbb{R}^{2}$ denoting an open bounded domain, and $T>0$ the time horizon. The control target is to match the given desired state $z$ in the $L^{2}(Q)$-sense by adjusting the body force $\mathcal{B} u$ in an appropriate manner. Above, $\mathcal{B}$ denotes an abstract control extension operator which maps controls of an abstract Hilbert space $\mathcal{U}$ to admissible right-hand sides of the Navier-Stokes system (D). In this context the method of instantaneous control serves a dual purpose - to construct a closed loop feedback control law which steers the system state $y$ to $z$ for $t$ tending to $\infty$, and to compute open loop control policies which (hopefully) approximate optimal open loop control strategies, i.e solutions of

$$
\left\{\begin{array}{l}
\min J(y, u)=\frac{1}{2} \int_{0}^{T} \int_{\Omega}|y-z|^{2} d x d t+\frac{\alpha}{2} \int_{0}^{T}|u|_{\mathcal{U}}^{2} d t \\
\text { s.t. (D). }
\end{array}\right.
$$

Optimal control problems for the Navier-Stokes system of the form of $(\mathbf{P})$ are mathematically and numerically well understood $[1,2,9,13]$. For $(\mathbf{P})$ Instantaneous control may be regarded as a suboptimal control approach which provides control policies on the time horizon $(0, T)$. However, 
as is shown in [14], instantaneous control may be regarded as as nonlinear feedback control policy and it is the the main aim of this work to extend the stability analysis presented there to the Navier-Stokes system.

Instantaneous control works as follows. The uncontrolled Navier-Stokes equations are discretized with respect to time. Then, at selected time slices an instantaneous version of the cost functional is approximately minimized w.r.t. a stationary quasi-Navier-Stokes system, whose structure depends on the chosen time-discretization method. The control obtained is used to steer the system to the next time slice, where the procedure is repeated.

Instantaneous control therefore is closely related to receding horizon control (rhc) or model predictive control (mpc) with finite time horizon [7, 21, 22]. It was applied to control the Burgers equation with stochastic forcing in [5] and also successfully to compute suboptimal controls for a great variety of fluid mechanical control problems $[3,4,10,12,19,20]$, and in the recent past also as real-time control approach to the cooling of steel [25]. Linear body force feedback control for the Navier-Stokes system is investigated in $[8,17,18]$.

As far as the author knows there are only few stability investigations for the application of the method to the control of infinite-dimensional systems [14, 16].

In the present work the results obtained in [14] are extended to the instationary Navier-Stokes system in two spatial dimensions. While for the continuous control policies developed in [14] similar stability properties can be proved as in the case of the Burgers equation, for the discrete controllers developed there only conditional stability can be shown for the Navier-Stokes system (Theorems $6,7)$. This motivates the construction of a slightly modified control policy, for which unconditional stability is proved in Theorem 8 . The main results of the present work can be summarized as follows.

\section{Main results:}

1. Given a sufficiently smooth desired state $z$, instantaneous control of the instationary NavierStokes system can be regarded as time discretization of a closed loop feedback policy $K$ which steers the system exponentially fast to $z$, i.e. with $S$ denoting the Stokes operator and $b(y)$ the nonlinearity of the Navier-Stokes equations

$$
y_{t}+\nu S y+b(y)=K(y)
$$

the solution of this system satisfying $|y(t)-z(t)|_{H^{1}} \leq c \exp (-\gamma t)$ with some positive constants $c$ and $\gamma$ (Theorems 4,5).

2. Instantaneous control may be regarded as a discrete-in-time feedback policy which steers the dynamical system exponentially fast to the desired state $z$, provided that either $z(0)$ is sufficiently close to the initial value $\phi$, or that the viscosity parameter $\nu$ is sufficiently large (Theorems 6,7 ). 3. Instantaneous control gives rise to a discrete-in-time feedback policy which steers the dynamical system exponentially fast to the desired state $z$ (Theorem 8).

The paper is organized as follows. In Section 2 an appropriate functional analytic framework is introduced and preliminary results are collected. In Section 3 the derivation of the instantaneous control approach, the formulation of the algorithm and its interpretation as nonlinear continuous and discrete-in-time feedback control policy is sketched. The results in this part of the work are similar to those in [14] and are stated for the convenience of the reader. In Section 4 both exponential stability of the continuous and conditonal exponential stability of the discrete controllers are shown. To obtain these results in consequence of the nonlinearity in the Navier-Stokes system a more subtle analysis is necessary than for the Burgers equation in [14]. Moreover, a slightly modified discrete controller is proposed for which unconditional exponential stability is proved. Finally, in Section 5 numerical examples are presented, which illustrate the theoretical results, and 
also compare the feedback policy on a time horizon $[0, T]$ to the corresponding optimal open-loop control strategy for $(\mathbf{P})$.

Throughout this work $c$ and $C$ denote global generic constants whose dependencies are mentioned when necessary.

\section{Preliminaries}

Set $V=\left\{v \in H_{0}^{1}(\Omega)^{2}, \operatorname{div} v=0\right\}, H=\cos _{L^{2}(\Omega)^{2}}\left\{v \in C_{0}^{\infty}(\Omega)^{2}\right.$, div $\left.v=0\right\}$ and identify the Hilbert space $H$ with its dual $H^{\prime}$. On $H$ the common inner product is used, and $V$ is endowed with the inner product

$$
(\varphi, \psi)_{V}=(\nabla \varphi, \nabla \psi)_{H} \text { for } \varphi, \psi \in V .
$$

Moreover, with $Z$ denoting a Hilbert space, $L^{p}(Z)(1 \leq p \leq \infty)$ denotes the space of measurable abstract functions $\varphi:(0, T) \rightarrow Z$, which are $p$-integrable $(1 \leq p<\infty)$, or essentially bounded on $(0, T)(p=\infty)$, respectively.

As control space $L^{2}(\mathcal{U})$ is taken, where $\mathcal{U}$ denotes the Hilbert space of abstract controls. The space $\mathcal{U}$ also is identified with its dual. Furthermore,

$$
\mathcal{B}: \mathcal{U} \rightarrow V^{\prime}
$$

denotes the control extension operator which is assumed to be bounded. In order to formulate the weak form of the instationary Navier-Stokes equations let $W:=W(V)=\left\{\varphi \in L^{2}(V): \varphi_{t} \in\right.$ $\left.L^{2}\left(V^{\prime}\right)\right\}$ supplied with the common inner product. Further, introduce

$$
b(u, v, w):=\int_{\Omega}(u \cdot \nabla) v w d x .
$$

Then from [24],

$$
b(u, v, w) \leq C \begin{cases}|u|_{H}^{\frac{1}{2}}|u|_{V}^{\frac{1}{2}}|v|_{H}^{\frac{1}{2}}|v|_{V}^{\frac{1}{2}}|w|_{V} & \forall u, v, w \in V \\ |u|_{H}^{\frac{1}{2}}|u|_{V}^{\frac{1}{2}}|v|_{V}^{\frac{1}{2}}|\Delta v|_{H}^{\frac{1}{2}}|w|_{H} & \forall u \in V, v \in V \cap H^{2}(\Omega)^{2}, w \in H \\ |u|_{H}|v|_{V}|w|_{H}^{\frac{1}{2}}|\Delta w|_{H}^{\frac{1}{2}} & \forall u \in H, v \in V, w \in V \cap H^{2}(\Omega)^{2} \\ |u|_{H}^{\frac{1}{2}}|\Delta u|_{H}^{\frac{1}{2}}|v|_{V}|w|_{H} & \forall u \in V \cap H^{2}(\Omega)^{2}, v \in V, w \in H,\end{cases}
$$

with a positive constant $C$, which for the uppermost estimate can be chosen as $C=\sqrt{2}[23$, Chap.III,(3.55)]. Moreover, for $y \in L^{2}(V)$ the function $b(y)$ defined by

$$
\langle b(y), v\rangle_{V^{\prime}, V}:=-b(y, y, v) \text { for all } v \in V
$$

is an element of $V^{\prime}$ for almost all $t \in(0, T)$ and $b(y) \in L^{1}(V)$. Now let $P: L^{2}(\Omega)^{2} \rightarrow H$ denote the Leray projector [6, Remark 1.10]. Then, the Stokes operator $S$ is given by

$$
S: \mathcal{D}(S) \subset H \rightarrow H, \quad S:=-P \Delta, \quad \mathcal{D}(S)=H^{2}(\Omega)^{2} \cap V .
$$

Further define

$$
A:=\nu S
$$

and denote by $B$ the solution operator of

$$
v+h A v=f \text { in } V^{\prime},
$$


where $f \in V^{\prime}$ is given. The operator $B$ is linear, bounded and self-adjoint and there holds $B^{-1}=$ $I+h A$. $V$,

In this setting the Navier-Stokes system (D) may be rewritten as Burgers equation in the space

$$
\begin{aligned}
& y_{t}+A y=b(y)+\mathcal{B} u, \\
& y(0)=\phi,
\end{aligned}
$$

where the nonlinearity $b(y)$ is defined in (3). The derivation and also the interpretation of instantaneous control for the Navier-Stokes system in the following therefore is abutted to the exposition in [14].

Let $X=W \times L^{2}(\mathcal{U})$ and $Y=L^{2}\left(V^{\prime}\right) \times H$. Introducing the operator $e: X \rightarrow Y$ by

$$
e(y, u)=\left(e_{1}(y, u), e_{2}(y, u)\right)=\left(y_{t}-\nu \Delta y-b(y)-\mathcal{B} u, y(0)-\phi\right)
$$

the Navier-Stokes system (D) can be expressed in the form $e(y, u)=0$ in $Y$, and the optimal control problem $(\mathbf{P})$ can be regarded as minimization problem with equality constraints:

$$
\text { minimize } J(y, u) \quad \text { s.t. } \quad e(y, u)=0 .
$$

Among other things, it is shown in [13] that this problem admits a solution $\left(y^{*}, u^{*}\right) \in X$, and that both $J$ and $e$ are infinitely continuously Fréchet-differentiable.

Young's inequality

$$
a b \leq \delta a^{2}+\frac{1}{4 \delta} b^{2} \quad \forall a, b \geq 0, \delta>0,
$$

and the following Lemmata are frequently used in the proofs of the main theorems.

Lemma 1. For $y \in V$ let $w:=B y$. Then $w \in V \cap H^{3}(\Omega)^{2}$ and $S w \in V$. Moreover,

$$
|w|_{H}^{2} \geq|y|_{H}^{2}-2 \nu h|y|_{V}^{2} .
$$

Further, let $z:=B((y \nabla) y)=B b(y)$. Then $z \in V$ and

$$
|z|_{H}^{2} \leq \frac{1}{2 \nu h}|y|_{H}^{2}|y|_{V}^{2} .
$$

Proof. By the definition of the operator $B$ the regularity claim for $w$ follows from [23, Chap. I, Prop. 2.3]. Thus, $S w \in H^{1}(\Omega)^{2}[6$, Remark 1.10] and, since $y \in V, S w \in V$. Therefore, $S w$ can be utilized as test function in the equation

$$
w+h A w=y .
$$

This gives

$$
|w|_{V}^{2}+\nu h|S w|_{H}^{2}=\int_{\Omega} \nabla w \nabla y d x \Rightarrow|w|_{V}^{2}+2 \nu h|S w|_{H}^{2} \leq|y|_{V}^{2} .
$$

Furthermore, using $y$ as test function the latter estimate leads to

$$
|y|_{H}^{2}=\int_{\Omega} y w+\nu h \nabla y \nabla w d x \leq \frac{1}{2}|y|_{H}^{2}+\frac{1}{2}|w|_{H}^{2}+h \nu|y|_{V}|w|_{V} \leq \frac{1}{2}|y|_{H}^{2}+\frac{1}{2}|w|_{H}^{2}+h \nu|y|_{V}^{2},
$$

which gives the first claim. 
To prove the second claim take $z$ as test function in the equation

$$
z+h A z=b(y)
$$

This gives, using the first estimate of (2) and Young' inequality (5) with $\delta=\nu h$,

$$
|z|_{H}^{2}+\nu h|z|_{V}^{2} \leq|b(y)|_{V^{\prime}}|z|_{V} \leq \sqrt{2}|y|_{H}|y|_{V}|z|_{V} \leq \frac{1}{2 \nu h}|y|_{H}^{2}|y|_{V}^{2}+\nu h|z|_{V}^{2},
$$

which completes the proof of the lemma.

Lemma 2. Let $y \in V, \kappa:=B y$ and let $\tau:=B \kappa$. Then

$$
\int_{\Omega} B y B S y d x=|y|_{V}^{2}-\nu h \int_{\Omega}(S \kappa+S \tau) S y d x
$$

and

$$
|S \tau|_{H}^{2},|S \kappa|_{H}^{2} \leq \frac{1}{4 \nu h}|y|_{V}^{2}
$$

Proof. The definition of $\kappa$ and $\tau$ implies $\kappa \in H^{3}(\Omega)^{2} \cap V, \tau \in H^{5}(\Omega)^{2} \cap V$. Moreover, $S \kappa$ and $S \tau$ are elements of $V$. Integration by parts gives the first part of the claim. To obtain the second claim, test the equation for $\kappa$ with $S \kappa$. This gives

$$
|\kappa|_{V}^{2}+\nu h|S \kappa|_{H}^{2} \leq|y|_{V}|\kappa|_{V} \leq\left\{\begin{array}{l}
\frac{1}{4}|y|_{V}^{2}+|\kappa|_{V}^{2} \\
\frac{1}{2}|y|_{V}^{2}+\frac{1}{2}|\kappa|_{V}^{2}
\end{array}\right.
$$

where for the upper estimate Young's inequality (5) with $\delta=1$, and for the lower estimate with $\delta=\frac{1}{2}$, was used. Since the same estimate holds with $\kappa$ replaced by $\tau$ and $y$ replaced by $\kappa$, and $|\kappa|_{V} \leq|y|_{V}$ by the lower estimate, the lemma is proved.

\section{Instantaneous control strategy}

For $m \in \mathbb{N}$ an equidistant discretization of the time interval $(0, T)$ is defined by $h=\frac{T}{m}$ and $t_{k}=k h$, $k=0,1, \ldots, m$. Instantaneous versions of the cost functional $J$ in $(\mathbf{P})$ are given by

$$
J^{k}: V \times \mathcal{U} \rightarrow \mathbb{R}, \quad(y, u) \mapsto \frac{1}{2}\left|y-z^{k}\right|_{H}^{2}+\frac{\alpha}{2}|u|_{\mathcal{U}}^{2}
$$

where

$$
z^{k}=\frac{1}{h} \int_{t_{k}-\frac{h}{2}}^{t_{k}+\frac{h}{2}} z(s, \cdot) d s
$$

and $z(t, \cdot)=0$ for $t>T$. Finally, for $k=1, \ldots, m$ and $i=1,2$ introduce the operators $e_{i}^{k}$ : $V \times \mathcal{U} \rightarrow V^{\prime}$ by

$$
e_{1}^{k}(y, u)=(I+h A) y-h b\left(y^{k-1}\right)-y^{k-1}-\mathcal{B} u,
$$

and for later purposes also

$$
e_{2}^{k}(y, u)=(I+h A) y-h b(y)-y^{k-1}-\mathcal{B} u,
$$

where $y^{k-1}$ denotes the state at the previous time slice. 
The instantaneous optimal control problem for the semi-implicit time integration is given by $\left(\mathbf{P}^{k}\right)$

$$
\text { minimize } J^{k}(y, u) \text { subject to } e_{1}^{k}(y, u)=0 \text { in } V^{\prime} \text {, }
$$

where $y^{0}=\phi$. The initial value $\phi$ now is required to be an element of the space $V$. For given $y^{k-1}$ a pair $\left(y^{k}, u^{k}\right)$ satisfies the subsidiary condition $e_{1}^{k}(y, u)=0$ in $V^{\prime}$ if and only if

$$
\left.\nu h\left(y^{k}, \varphi\right)_{V}+\left(y^{k}, \varphi\right)_{H}=\left(y^{k-1}, \varphi\right)_{H}+\left\langle\mathcal{B} u^{k}+h b\left(y^{k-1}\right), \varphi\right\rangle\right)_{V^{\prime}, V} \quad \forall \varphi \in V .
$$

Since $\phi \in V$ holds, the right-hand side in this linear equation defines a bounded linear functional on $V$. Thus, for every $u^{k} \in \mathcal{U}$ Eq. (10) admits a unique solution $y^{k} \in V$ which satisfies the a-priori estimate

$$
\left|y^{k}\right|_{V} \leq \frac{C}{\nu h}\left(\left|y^{k-1}\right|_{H}+h\left|y^{k-1}\right|_{V}^{2}+\left|u^{k}\right|_{\mathcal{U}}\right) .
$$

Since $J^{k}$ is quadratic and $e_{1}^{k}$ is linear, every problem $\left(\mathbf{P}^{k}\right), k=1, \ldots, m$, admits a unique solution $\left(y_{*}^{k}, u_{*}^{k}\right) \in V \times \mathcal{U}$ which in fact defines a minimum for $\left(\mathbf{P}^{k}\right)$. Furthermore, the unique Lagrange multiplier $\lambda_{*}^{k} \in V$ together with the solution $\left(y_{*}^{k}, u_{*}^{k}\right)$ satisfies the first-order necessary optimality conditions (note that $A$ is selfadjoint)

$$
\begin{aligned}
(I+h A) y & =\mathcal{B} u+y^{k-1}+h b\left(y^{k-1}\right) \\
(I+h A) \lambda & =-\left(y-z^{k}\right) \\
\alpha u-\mathcal{B}^{\star} \lambda & =0
\end{aligned}
$$

The optimal control problem $\left(\mathbf{P}^{k}\right)$ is equivalent to the unconstrained minimization of the functional

$$
\hat{J}^{k}(u)=J^{k}(y(u), u)
$$

over $\mathcal{U}$, where for a control $u \in \mathcal{U}$ the state $y(u) \in V$ is given as the unique solution to (10). The gradient of $\hat{J}^{k}$ at $u$ is given by

$$
\nabla \hat{J}^{k}(u)=\alpha u-\mathcal{B}^{\star} \lambda
$$

where for given $u$ the function $\lambda$ is obtained by first solving the linear quasi-Stokes problem (11a) for the state $y$, and then solving (11b) for $\lambda$.

Remark 1. If one would use implicit time integration in problem $\left(\mathbf{P}^{k}\right)$, i.e. in the subsidiary condition the operator $e_{1}^{k}$ is replaced by $e_{2}^{k}$, the adjoint equation (11b) alters to

$$
(I+h A) \lambda-b^{\prime}(y)^{\star} \lambda=-\left(y-z^{k}\right) .
$$

Into this equation the state $y$ enters in two different fashions; firstly as observation $-\left(y-z^{k}\right)$, and secondly as coefficient in the nonlinearity $b^{\prime}(y)^{\star}$. Since the gradient $\nabla \hat{J}^{k}(u)$ at a control $u$ depends on $\lambda$ it in the present case depends on the observation $y^{k}-z^{k}$, and also on the whole state $y^{k}$ in terms of the derivative of $b$. This structure retains also in the case of boundary observation, where the observation enters as boundary condition into the adjoint equation, but the whole state $y^{k}$ again as a coefficient function. As a consequence, computation of gradient information for $\hat{J}^{k}$ in the present case can not be based on observations alone.

On the other hand the adjoint equation (11b) only depends on the observation $y^{k}-z^{k}$. Therefore, gradient information for the functional $\hat{J}^{k}$ is available utilizing the observations only. In the particular case of boundary observation no information of the state in the whole computational domain is needed at all. 


\subsection{The algorithm}

Instead of solving $\left(\mathbf{P}^{k}\right)$ exactly the instantaneous control strategy only provides approximate solutions to this problem through applying one step of the steepest descent method with stepsize $\rho>0$. The control obtained in this way is used to steer the system to the next time slice. With the gradient of the functional $\hat{J}^{k}$ available this procedure in algorithmical form can be formulated as follows.

\section{Algorithm 1 (Instantaneous control).}

1.) Set $y^{0}=\phi, k=0$ and $t^{0}=0$.

2.) Given an initial control $u_{0}^{k}$, solve

$$
\begin{aligned}
(I+h A) y & =y^{k}+h b\left(y^{k}\right)+\mathcal{B} u_{0}^{k}, \\
(I+h A) \lambda & =-\left(y-z^{k}\right) .
\end{aligned}
$$

3.) $\operatorname{Set} \nabla \hat{J}\left(u_{0}^{k}\right)=\alpha u_{0}^{k}-\mathcal{B}^{\star} \lambda$.

4.) Given $\rho>0$, set $u^{k+1}=u_{0}^{k}-\rho \nabla \hat{J}\left(u_{0}^{k}\right)$.

5.) Solve

$$
(I+h A) y^{k+1}=y^{k}+h b\left(y^{k}\right)+\mathcal{B} u^{k+1} .
$$

6.) Set $t_{k+1}=t_{k}+h, k=k+1$. If $t_{k}<T$ goto 2 .

Here, $\hat{J}=\hat{J}^{k}$. The choice of the stepsize $\rho$ in step 4.) of the algorithm is crucial. Since $\left(\mathbf{P}^{k}\right)$ is quadratic with linear constraints, the optimal choice $\rho^{*}$ can be computed exactly and in the present situation is given by

$$
\rho^{*}=-\frac{\left.(y(u)-z, y(d))_{H}+\alpha(u, d)\right)_{\mathcal{U}}}{|y(d)|_{H}^{2}+\alpha|d|_{\mathcal{U}}^{2}}=\frac{|d|_{\mathcal{U}}^{2}}{|y(d)|_{H}^{2}+\alpha|d|_{\mathcal{U}}^{2}} \leq \frac{1}{\alpha},
$$

where $d=-\nabla \hat{J}(u)$. The computation of $\rho^{*}$ requires only the computation of the auxiliary function $y(d)$.

It is shown in $[11,14]$ that Algorithm 1 allows the interpretation as a non-linear discrete-intime suboptimal closed loop control method, which turns out to be the stable time discretization of some continuous closed loop controller. For the convenience of the reader these results for the Navier-Stokes system are summarized in the following subsection. To simplify the exposition from now onwards $\mathcal{U}=V^{\prime}$ and thus, $\mathcal{B}=I$ is assumed. This choice is justified by the fact that in many applications of distributed control applied to systems governed by parabolic equations, the operator $\mathcal{B}$ defined in (1) plays the role of an extension operator.

\subsection{Feedback control laws}

It is shown in [11, Theorem 5.4.1], [14, Theorem 3] that Algorithm 1 allows the following interpretation.

\section{Theorem 3.}

i. For $u_{0}^{k}=0$ Algorithm 1 is equivalent to the semi-implicit time discretization with discretization step size $h$

$$
(I+h A) y^{k+1}=y^{k}+h b\left(y^{k}\right)-\rho B B\left(y^{k}-z^{k}\right)-h \rho B B\left(b\left(y^{k}\right)-A z^{k}\right), \quad y^{0}=\phi,
$$


of the dynamical system

$$
\dot{y}+A y-b(y)=K(y) \quad \text { in } L^{2}\left(V^{\prime}\right) \text { and } \quad y(0)=\phi
$$

where

$$
K(y)=-\frac{\rho}{h} B B(y-z)-\rho B B(b(y)-A z)
$$

ii. Choosing the initial control $u_{0}^{k}$ in Algorithm 1 as solution of

$$
\left(I-\frac{\rho}{1-\rho \alpha} B B\right) u_{0}^{k}=\frac{1}{1-\rho \alpha}\left(z^{k+1}-z^{k}+A z^{k+1}-b\left(z^{k}\right)+\rho B B\left(b\left(z^{k}\right)-A z^{k}\right)\right)
$$

the algorithm is equivalent to

$$
\begin{aligned}
(I+h A) w^{j+1}=w^{j}+h\left(b\left(y^{j}\right)-b\left(z^{j}\right)\right) & -\rho B B w^{j} \\
& -\rho h B B\left(b\left(y^{j}\right)-b\left(z^{j}\right)\right), \quad w^{0}=\phi-z(0),
\end{aligned}
$$

where $w:=y-z$. The related continuous system is given by

$$
y_{t}+A y-b(y)=K(y) \quad \text { in } L^{2}\left(V^{\prime}\right) \text { and } \quad y(0)=\phi
$$

where

$$
K(y)=-\frac{\rho}{h} B B(y-z)-\rho B B(b(y)-b(z))+z_{t}+A z-b(z) .
$$

It is now clear that the nonlinear operators $K$ defined in (16) and (19), respectively can be interpreted as non-linear closed-loop control policies for the Navier-Stokes equations. In this context it is important to note that the discretization step-size $h$ and the descent parameter $\rho$ in the gradient step of Algorithm 1 in the continuous case may now be regarded as parameters defining the controller.

The discrete counterpart to (19) will frequently be used in the sequel. It is given by

$$
K^{D}(y)=-\frac{\rho}{h} B B\left(y-z^{j}\right)-\rho B B\left(b(y)-b\left(z^{j}\right)\right)+\frac{z^{j+1}-z^{j}}{h}+A z^{j+1}-b\left(z^{j}\right) .
$$

Unless otherwise stipulated, from now onwards

Assumption 3.1. $0 \neq \phi \in V$ and $z \in H^{2,1}(Q)$.

Note that this assumption on the desired state $z$ in particular implies that $z(0)$ is meaningful. Moreover, $z(0) \in V$.

\section{Existence, uniqueness and stability of solutions}

In this section existence, uniqueness, stability and regularity of a solution to (18). The boundary $\partial \Omega$ is assumed to be as smooth as required by the existence and regularity results for the Stokes and quasi-Stokes problems considered in the proofs of the Theorems below, see [23, Chap. I, Prop. 2.3]. It follows from the a-priori estimates to be derived that the stabilized system (18) admits a unique solution. Moreover, the $H$ and $V$ norms of difference $w=y-z$ decay exponentially with rate $-\frac{\rho}{h}$. To achieve these results the range of $\rho$ has to be adapted to the size of $|z|_{L^{\infty}(H)}^{2}$. For this purpose set $d_{H}:=2|\phi-z(0)|_{H}^{2}$ and let the range of $\rho$ be implicitly defined by

$$
0<\rho \leq \rho_{1}:=\min \left(\rho_{0}, \frac{\nu^{2}}{2 \nu^{2}+\exp \left(\frac{4+\rho}{\nu}|z|_{L^{2}(V)}^{2}\right) d_{H}+|z|_{L^{\infty}(H)}^{2}}\right) \text {. }
$$


Theorem 4. Let $0<\rho \leq \rho_{1}$ with $\rho_{1}$ from (21) and $h>0$ be fixed. Further, let $\phi \in H$ and $z \in W$. Then (18) for every $T>0$ admits a unique solution $y \in W$ and the difference $w=y-z$ satisfies

$$
\left\{\begin{array}{l}
|w|_{H}^{2} \leq C\left(\nu,|z|_{W}\right) \mathrm{e}^{-\frac{\rho}{h} t} \quad \text { for all } t \in[0, T] \\
|w|_{L^{\infty}(H)}^{2} \leq C\left(\nu,|z|_{W}\right), \\
|w|_{L^{2}(V)}^{2} \leq C\left(\nu,|z|_{W}\right) \\
\left|w_{t}\right|_{L^{2}\left(V^{\prime}\right)}^{2} \leq C\left(\nu,|z|_{W}\right)\left\{1+\frac{\rho}{h}\right\}
\end{array} \quad\right. \text { and }
$$

where $C\left(\nu,|z|_{W}\right)$ is a positive constant independent of $\rho$ and $h$.

Proof. Existence of a solution can be proved using a Galerkin Ansatz in combination with the estimates derived below, compare $[11,14]$. Uniqueness also follows from these estimates. To prove (22) use $w$ as test function in the variational formulation of (18). This leads to

$$
\begin{aligned}
\frac{\mathrm{d}}{\mathrm{dt}}|w|_{H}^{2}+\nu|w|_{V}^{2}+\frac{\rho}{h}|B w|_{H}^{2}= & \\
& \int_{\Omega}((w \nabla) w+(z \nabla) w+(w \nabla) z) w d x \\
& -\rho \int_{\Omega}(B((w \nabla) w+(z \nabla) w+(w \nabla) z)) B w d x=(i)+\cdots+(v i) .
\end{aligned}
$$

Since $(i)=(i i)=0$, it remains to estimate the terms $(i i i),(i v),(v)$ and $(v i)$ in order to derive a differential inequality for $y-z$. To begin with, estimate, using Young's inequality and (7),

$$
\begin{gathered}
(i i i) \leq \frac{\nu}{2}|w|_{V}^{2}+\frac{2}{\nu}|z|_{V}^{2}|w|_{H}^{2}, \\
(i v) \leq \rho h|B((w \nabla) w)|_{H}^{2}+\frac{\rho}{4 h}|B w|_{H}^{2} \leq \frac{\rho}{2 \nu}|w|_{H}^{2}|w|_{V}^{2}+\frac{\rho}{4 h}|B w|_{H}^{2}, \\
(v)+(v i) \leq \rho h\left\{|B((w \nabla) z)|_{H}^{2}+|B((z \nabla) w)|_{H}^{2}\right\} \\
\leq \frac{\rho}{4 h}|B w|_{H}^{2} \\
\leq \frac{\rho}{4 h}|B w|_{H}^{2}+\frac{\rho}{2 \nu}\left\{|w|_{V}^{2}|z|_{H}^{2}+|z|_{V}^{2}|w|_{H}^{2}\right\},
\end{gathered}
$$

and apply estimate (6). This leads to

$$
\frac{1}{2} \frac{d}{d t}|w|_{H}^{2}+\left(\nu\left(\frac{1}{2}-\rho\right)-\frac{\rho}{2 \nu}\left(|w|_{H}^{2}+|z|_{L^{\infty}(H)}^{2}\right)\right)|w|_{V}^{2}+\frac{\rho}{2 h}|w|_{H}^{2} \leq \frac{4+\rho}{2 \nu}|z|_{V}^{2}|w|_{H}^{2} .
$$

Since $\rho$ satisfies $(21), \nu\left(\frac{1}{2}-\rho\right)-\frac{\rho}{2 \nu}\left(\exp \left(\frac{4+\rho}{\nu}|z|_{L^{2}(V)}^{2}\right) d_{H}+|z|_{L^{\infty}(H)}^{2}\right)>0$. For $\rho$ in this range standard arguments yield

$$
\text { (23) } \begin{aligned}
\frac{1}{2} \frac{d}{d t}|w|_{H}^{2}+\left(\nu\left(\frac{1}{2}-\rho\right)-\frac{\rho}{2 \nu}\left(\exp \left(\frac{4+\rho}{\nu}|z|_{L^{2}(V)}^{2}\right) d_{H}+|z|_{L^{\infty}(H)}^{2}\right)\right) & |w|_{V}^{2}+\frac{\rho}{2 h}|w|_{H}^{2} \\
& \leq \frac{4+\rho}{2 \nu}|z|_{V}^{2}|w|_{H}^{2}
\end{aligned}
$$

Since the right-hand-side in (23) is integrable a further Gronwall argument gives the desired result. Note that the estimate for $w_{t}$ is a direct consequence of the second and third estimate in (22). 
A similar result holds for the decay w.r.t. the $V$-norm of $w$. Let now the range of $\rho$ implicitly defined by the relation

$$
0<\rho \leq \rho_{2}:=\min \left(\rho_{0}, \frac{1}{2} \frac{\nu^{2}}{2 \nu^{2}+3 \exp \left(\frac{4+\rho}{\nu}|z|_{L^{2}(V)}^{2}\right) d_{H}+3|z|_{L^{\infty}(H)}^{2}}\right)
$$

Theorem 5. Let $\rho$ satisfy $(24)$ and let $y$ be the unique solution of (18). Then $y \in H^{2,1}(Q)$ and $w=y-z$ satisfies

$$
\begin{array}{lll}
|w|_{V}^{2} & \leq C\left(\nu,|z|_{H^{2,1}(Q)}\right) \mathrm{e}^{-\frac{\rho}{h} t} \quad \text { for all } t \in[0, T] \\
|w|_{L^{\infty}(V)}^{2} & \leq C\left(\nu,|z|_{H^{2,1}(Q)}\right), \\
|w|_{L^{2}\left(H^{2}(\Omega)^{2} \cap V\right)}^{2} & \leq C\left(\nu,|z|_{H^{2,1}(Q)}\right) \\
\left|w_{t}\right|_{L^{2}(H)}^{2} & \leq C\left(\nu,|z|_{H^{2,1}(Q)}\right)\left\{1+\frac{\rho}{h}\right\},
\end{array} \quad \text { and }
$$

where $C\left(\nu,|z|_{H^{2,1}(Q)}\right)$ is a positive constant independent of $\rho$ and $h$.

Proof. Use $S w$ as test function in (18). This leads to

$$
\begin{aligned}
\frac{1}{2} \frac{d}{d t}|w|_{V}^{2}+\nu|S w|_{H}^{2}+\int_{\Omega}((y \nabla) y-(z \nabla) z) S w d x= \\
\quad-\frac{\rho}{h} \int_{\Omega} B w B S w d x-\rho \int_{\Omega} B((y \nabla) y-(z \nabla) z) B S w d x
\end{aligned}
$$

Relation (24) implies $1-\rho-\frac{3 \rho}{\nu^{2}}\left(\exp \left(\frac{4+\rho}{\nu}|z|_{L^{2}(V)}^{2}\right) d_{H}+|z|_{L^{\infty}(H)}^{2}\right) \geq \frac{1}{2}$. Restricting $\rho$ to this range, similar to the derivation of (23) after several applications of (2), (8), Lemma 2, and Young's inequality one ends up with

$$
\begin{aligned}
\frac{1}{2} \frac{d}{d t}|w|_{V}^{2}+\frac{\nu}{2}|S w|_{H}^{2}+\frac{\rho}{h}\left(1-\rho-\frac{3 \rho}{\nu^{2}}\right. & \left.\left(\exp \left(\frac{4+\rho}{\nu}|z|_{L^{2}(V)}^{2}\right) d_{H}+|z|_{L^{\infty}(H)}^{2}\right)\right)|w|_{V}^{2} \\
& \leq C_{\nu}\left\{|w|_{H}^{2}|w|_{V}^{2}+|z|_{H}|S z|_{H}+|z|_{V}^{2}+|z|_{V}^{4}\right\}|w|_{V}^{2}
\end{aligned}
$$

Since the right-hand-side in (25) is integrable a Gronwall argument gives the desired result.

\subsection{Stability of discrete controllers}

Here, firstly the stability properties of the instantaneous control procedure (17) are investigated. As will be shown stability for a certain parameter range of $h$ and $\rho$ can only be ensured by requiring additionally either largeness of the viscosity parameter $\nu$ or smallness of $\phi-z(0)$. As is shown in [14] these restrictions do not apply when the procedure is applied to the instationary Burgers equation. Secondly, a slightly modified version of the controller (20) is applied to the fully implicit Euler-discretization of the Navier-Stokes system. It turns out that the resulting discrete-in-time system for $w=y-z$ is unconditional stable. Note, that fully implicit discretization of the state is a realistic situation, since the discrete controller is applied to stabilize a physical system which is described by the Navier-Stokes equations. Therfore, the choice of the discretization procedure for the uncontrolled state need not be linked to the discrete controller.

Throughout this section it is assumed that 
Assumption 4.1. In addition to Assumption 3.1 let $z \in C\left([0, T] ; H^{1, \infty}(\Omega)^{2} \cap V\right)$.

In a preparatory step an inequality relating the $H$-norms of $w=y^{j+1}-z^{j+1}$ and $v=y^{j}-z^{j}$ is derived, and $z:=z^{j}, j \in \mathbb{N}$. To begin with test (17) with $w$. This gives

(26) $\frac{1}{2}|w|_{H}^{2}-\frac{1}{2}|v|_{H}^{2}+\frac{1}{2}|w-v|_{H}^{2}+\nu h|w|_{V}^{2}=$

$$
\begin{aligned}
& h \int_{\Omega}\left((v \nabla) v+\left(z^{j} \nabla\right) v+(v \nabla) z^{j}\right) w d x-\rho \int_{\Omega} B v B w d x \\
& \quad-\rho h \int_{\Omega} B\left((v \nabla) v+\left(z^{j} \nabla\right) v+(v \nabla) z^{j}\right) B w d x=(i)+\cdots+(v i i) .
\end{aligned}
$$

Estimation, using (2),

$$
|B((v \nabla) v)|_{H}^{2} \leq \frac{1}{2 \nu h}|v|_{H}^{2}|v|_{V}^{2}
$$

from Lemma 1, and Young's inequality, yields

(i) $\quad \leq \frac{\nu h}{4}|w|_{V}^{2}+\frac{2 h}{\nu}|v|_{H}^{2}|v|_{V}^{2}$,

(ii) $\leq \frac{\nu h}{4}|w|_{V}^{2}+\frac{|z|_{\infty}^{2} h}{\nu}|v|_{H}^{2}$,

(iii) $\leq \frac{h}{2}|w|_{H}^{2}+\frac{|z|_{1, \infty}^{2} h}{2}|v|_{H}^{2}$,

(iv) $=-\rho \int_{\Omega}|B w|^{2} d x-\rho \int_{\Omega} B(v-w) B w \leq \frac{\rho}{2}(\rho-1)|B w|_{H}^{2}+\frac{1}{2}|v-w|_{H}^{2}$,

$(v) \quad=-\rho h \int_{\Omega} B((v \nabla) v) B w d x \leq \frac{3 \rho h}{2 \nu(1-\rho)}|v|_{H}^{2}|v|_{V}^{2}+\frac{\rho}{12}(1-\rho)|B w|_{H}^{2}$,

and finally

$$
\begin{aligned}
(v i)+(v i i)=-\rho h \int_{\Omega} B\left((v \nabla) z^{j}+\left(z^{j} \nabla\right) v\right) B w d x & \\
& \leq\left\{\frac{3 \rho h^{2}|z|_{1, \infty}^{2}}{1-\rho}+\frac{3 \rho h|z|_{\infty}^{2}}{4(1-\rho) \nu}\right\}|v|_{H}^{2}+\frac{\rho(1-\rho)}{6}|B w|_{H}^{2} .
\end{aligned}
$$

Now introduce

$$
c_{1}(\rho, h):=\frac{1}{2}+\frac{\rho}{4}(1-\rho)-\frac{h}{2},
$$

and

$$
c_{2}^{j}(\rho, h, z):=\frac{1}{2}+\left[\frac{2 h}{\nu}+\frac{3 h \rho}{2 \nu(1-\rho)}\right]|v|_{V}^{2}+\underbrace{\left[\frac{|z|_{1, \infty}^{2} h}{2}+\frac{|z|_{\infty}^{2} h}{\nu}+\frac{3 \rho h^{2}|z|_{1, \infty}^{2}}{1-\rho}+\frac{3 \rho h|z|_{\infty}^{2}}{(1-\rho) 4 \nu}\right]}_{=: \tilde{c}_{2}(h, \rho, z)} .
$$

With this notation the estimates above and Lemma 1 together with (26) give

$$
c_{1}(\rho, h)|w|_{H}^{2}-c_{2}^{j}(\rho, h, z)|v|_{H}^{2}+\frac{\nu h}{2}\left(1-\rho+\rho^{2}\right)|w|_{V}^{2} \leq 0 .
$$

Theorem 6. (Conditional $H$-norm stability of instantaneous control) Let $w^{j}:=y^{j}-z^{j}$, where $y^{j}$ denotes the iterates obtained by (17).

$$
{ }_{\rho} \rho(0,1){ }^{\exists} h^{*}(\rho), 0<\kappa<1{ }^{\forall} j \in \mathbb{N}:\left|w^{j+1}\right|_{H}^{2} \leq \kappa^{j}\left|w^{0}\right|_{H}^{2},
$$


provided $0<h \leq h^{*}(\rho)$ and

$$
\text { crit }:=\frac{4-\rho}{\nu^{2}(1-\rho)\left(1-\rho+\rho^{2}\right)}\left|w^{0}\right|_{H}^{2}
$$

is sufficiently small.

Proof. Fix $\rho \in(0,1)$, define

$$
\hat{c}_{2}(\rho, h, z):=\frac{1}{2}+\operatorname{crit}+\tilde{c}_{2}(\rho, h, z)
$$

and argue by induction as follows.

1. Set $j=0$, choose $h_{0}=h_{0}(\rho)$ and crit so small that for all $0<h \leq h_{0}$

(a) $\hat{c}_{2}(\rho, h, z) \leq 1$ and $c_{2}^{0}(\rho, h, z) \leq 1$,

(b) $\frac{\hat{c}_{2}(\rho, h, z)}{c_{1}(\rho, h)}=\kappa_{1}<1$ and

(c) $\frac{c_{2}^{0}(\rho, h, z)}{c_{1}(\rho, h)}=\kappa_{2}<1$

holds. This is possible, since the for $\rho \in(0,1)$ the term $\frac{\rho}{4}(1-\rho)$ in the definition of $c_{1}(\rho, h)$ is positive. Define $\kappa:=\max \left(\kappa_{1}, \kappa_{2}\right)$. Then (27) implies

$$
\left|w^{1}\right|_{H}^{2} \leq \kappa\left|w^{0}\right|_{H}^{2}
$$

and

$$
\left|w^{1}\right|_{V}^{2} \leq \frac{2}{\nu h\left(1-\rho+\rho^{2}\right)}\left|w^{0}\right|_{H}^{2}=\frac{2}{\nu h\left(1-\rho+\rho^{2}\right)} \kappa^{0}\left|w^{0}\right|_{H}^{2} .
$$

2. Now assume that for $j \in \mathbb{N}$

(a) $\left|w^{j}\right|_{H}^{2} \leq \kappa^{j}\left|w^{0}\right|_{H}^{2}$ and

(b) $\left|w^{j}\right|_{V}^{2} \leq \frac{2}{\nu h\left(1-\rho+\rho^{2}\right)} \kappa^{j-1}\left|w^{0}\right|_{H}^{2}$

holds true.

3. Then conclude from (27)

$$
\begin{aligned}
c_{2}^{j}(\rho, h, z) & =\frac{1}{2}+\left[\frac{2 h}{\nu}+\frac{3 h \rho}{2 \nu(1-\rho)}\right]\left|w^{j}\right|_{V}^{2}+\tilde{c}_{2}(\rho, h, z) \\
& \leq \frac{1}{2}+\left[\frac{2 h}{\nu}+\frac{3 h \rho}{2 \nu(1-\rho)}\right] \frac{2}{\nu h\left(1-\rho+\rho^{2}\right)} \kappa^{j-1}\left|w^{0}\right|_{H}^{2}+\tilde{c}_{2}(\rho, h, z) \\
& \leq \frac{1}{2}+\operatorname{crit}+\tilde{c}_{2}(\rho, h, z)=\hat{c}_{2}(\rho, h, z) .
\end{aligned}
$$

Thus, a further application of (27) implies

$$
\left|w^{j+1}\right|_{H}^{2} \leq \frac{c_{2}^{j}(\rho, h, z)}{c_{1}(\rho, h)}\left|w^{j}\right|_{H}^{2} \leq \frac{\hat{c}_{2}(\rho, h, z)}{c_{1}(\rho, h)}\left|w^{j}\right|_{H}^{2} \leq \kappa^{j+1}\left|w^{0}\right|_{H}^{2}
$$

and

$$
\left|w^{j+1}\right|_{V}^{2} \leq \frac{2}{\nu h\left(1-\rho+\rho^{2}\right)} c_{2}^{j}(\rho, h, z)\left|w^{j}\right|_{H}^{2} \leq \frac{2}{\nu h\left(1-\rho+\rho^{2}\right)} \kappa^{j}\left|w^{0}\right|_{H}^{2},
$$


which completes the proof of Theorem 6 .

The last estimate of the previous proof also yields stability with respect to the $V$ norm.

Theorem 7. (Conditional $V$-norm stability of instantaneous control)

$$
\forall \rho \in(0,1){ }^{\exists} h^{*}(\rho), 0<\kappa<1{ }^{\forall} j \in \mathbb{N}, 0<h \leq h^{*}:\left|w^{j}\right|_{V} \leq \frac{2}{\nu h\left(1-\rho+\rho^{2}\right)} \kappa^{j-1}\left|w^{0}\right|_{H},
$$

provided

$$
\text { crit }:=\frac{4-\rho}{\nu^{2}(1-\rho)\left(1-\rho+\rho^{2}\right)}\left|w^{0}\right|_{H}^{2}
$$

is sufficiently small.

Remark 2. The smallness of crit in Theorems 6 and 7 is a condition either on the smallness of the initial difference between state and desired state or on the smallness on the Reynolds number of the fluid. It has to be required since there are no better estimates available for the term $(i)$ in (26). The term $(v)$ in $(26)$ could be estimated in a slightly different way to obtain a $\rho^{2}$ in front of $|v|_{V}^{2}$, see the proof of the next Theorem, and therefore could be reduced by decreasing $\rho$. However, for $(i)$ in (26) there is no further knob to fix its size. For the Burgers equation the situation is much more comfortable at this stage. Due to the continuous embedding $H^{1} \hookrightarrow L^{\infty}$ and the well known $L^{2}-H^{1}$ interpolation estimate for $L^{\infty}$ functions in one spatial dimension one has

$$
h \int_{\Omega} v v^{\prime} w d x \leq \frac{\nu h}{4}|w|_{V}^{2}+h^{1-2 \alpha}|w|_{H}^{2}+h^{1+2 \alpha} \frac{1}{2 \sqrt{\nu}}|w|_{V}^{2}|w|_{H}^{2} \quad \text { for all } \alpha \in(0,1) .
$$

Following the lines of the proof of Theorem 6 one can now conclude that the smallness requirement on crit may be dropped provided $\rho$ is sufficiently small, since the power of $h$ in the last addend on the right-hand-side of this estimate is larger than one. For more details see [14].

Finally, a discrete-in-time control policy for the Navier-Stokes system is considered which is unconditional stable. Let

$$
K^{D}(y)=-\frac{\rho}{h} B B\left(y-z^{j}\right)-\rho B B\left(b(y)-b\left(z^{j}\right)\right)+\frac{z^{j+1}-z^{j}}{h}+A z^{j+1}-b\left(z^{j+1}\right),
$$

and consider the following discretization of (18);

$$
\frac{y^{j+1}-y^{j}}{h}+A y^{j+1}-b\left(y^{j+1}\right)=K^{D}\left(y^{j}\right), \quad j=0,1, \ldots \quad \text { and } y^{0}=\phi .
$$

There holds

Theorem 8. ( $H$ - and $V$-norm stability of $(28))$

Let $w^{j}:=y^{j}-z^{j}$. There exists some $\rho^{*} \in(0,1)$ such that for every $0<\rho \leq \rho^{*}$ there exists a $h^{*}(\rho)>0$ and a positive $\kappa<1$ such that for all $j \in \mathbb{N}$

$$
\begin{aligned}
\left|w^{j}\right|_{H}^{2} & \leq \kappa^{j}\left|w^{0}\right|_{H}^{2} \quad \text { and } \\
\left|w^{j}\right|_{V}^{2} & \leq \frac{2}{\nu h\left(1-\frac{2}{3} \rho+\frac{2}{3} \rho^{2}\right)} \kappa^{j-1}\left|w^{0}\right|_{H}^{2},
\end{aligned}
$$

provided $0<h \leq h^{*}(\rho)$. 
Proof. During the proof again let $v=y^{j}-z^{j}, w=y^{j+1}-z^{j+1}$ and test (29) with $w$. This leads to

$$
\begin{gathered}
\text { (30) } \frac{1}{2}|w|_{H}^{2}-\frac{1}{2}|v|_{H}^{2}+\frac{1}{2}|w-v|_{H}^{2}+h \nu|w|_{V}^{2}= \\
h \int_{\Omega}\left((w \nabla) w+\left(z^{j} \nabla\right) w+(w \nabla) z^{j}\right) w d x-\rho \int_{\Omega} B v B w d x \\
-\rho h \int_{\Omega} B\left((v \nabla) v+\left(z^{j} \nabla\right) v+(v \nabla) z^{j}\right) B w d x=(i)^{\prime}+(i i)^{\prime}+(i i i)^{\prime}+(i v)+(v)^{\prime}+(v i)+(v i i)
\end{gathered}
$$

where $(i v),(v i)$ and $(v i i)$ are defined in $(26)$. There holds $(i)^{\prime}=(i i)^{\prime}=0$ and $(i i i)^{\prime}$ can be estimated as

$$
(i i i)^{\prime} \leq \frac{\nu h}{2}|w|_{V}^{2}+\frac{h}{\nu}|z|_{V}^{2}|w|_{H}^{2}
$$

Utilizing the estimate $|B B w|_{V} \leq|w|_{V}$ one obtains

$$
(v)^{\prime} \leq \frac{\nu h}{3}|w|_{V}^{2}+\frac{3 \rho^{2} h}{2 \nu}|v|_{H}^{2}|v|_{V}^{2}
$$

The remaining addenda can be estimated as above. Now introduce

$$
c_{1}(\rho, h, z):=\frac{1}{2}+\frac{\rho}{3}(1-\rho)-\frac{2 h}{\nu}|z|_{L^{\infty}(V)}^{2}-\frac{h}{2},
$$

and

$$
c_{2}^{j}(\rho, h, z):=\frac{1}{2}+\frac{3 \rho^{2} h}{2 \nu}|v|_{V}^{2}+\underbrace{\left[\frac{3 \rho h^{2}|z|_{1, \infty}^{2}}{1-\rho}+\frac{3 \rho h|z|_{\infty}^{2}}{4(1-\rho) \nu}\right]}_{=: \tilde{c}_{2}(h, \rho, z)} .
$$

With this notation and the estimates above one concludes from (30)

$$
c_{1}(\rho, h, z)|w|_{H}^{2}-c_{2}^{j}(\rho, h, z)|v|_{H}^{2}+\frac{\nu h}{2}\left(1-\frac{2}{3} \rho+\frac{2}{3} \rho^{2}\right)|w|_{V}^{2} \leq 0 .
$$

Now define

$$
\hat{c}_{2}(\rho, h, z):=\frac{1}{2}+\frac{3 \rho^{2}}{\nu^{2}\left(1-\frac{2}{3} \rho+\frac{2}{3} \rho^{2}\right)}\left|w^{0}\right|_{H}^{2}+\tilde{c}_{2}(\rho, h, z)
$$

and proceed as follows.

1. Choose $\rho^{*} \in(0,1)$ such that

$$
\frac{3 \rho}{\nu^{2}\left(1-\frac{2}{3} \rho+\frac{2}{3} \rho^{2}\right)}\left|w^{0}\right|_{H}^{2} \leq \frac{1}{6}(1-\rho) \quad \text { for all } \rho \in\left(0, \rho^{*}\right] .
$$

2. Fix $\rho \in\left(0, \rho^{*}\right]$ and choose $h^{*}=h^{*}(\rho)>0$ such that
(a) $\tilde{c}_{2}(h, \rho, z) \leq \frac{1}{4}$
(b) $\frac{3 \rho^{2} h}{2 \nu}\left|w^{0}\right|_{V}^{2} \leq \frac{1}{4}$
(c) $\frac{h}{2}+\frac{2 h}{\nu}|z|_{L^{\infty}(V)}^{2}+\tilde{c}_{2}(h, \rho, z)<\frac{\rho}{6}(1-\rho)$
(d) $\frac{c_{2}^{0}(\rho, h, z)}{c_{1}(\rho, h, z)} \leq \kappa_{1}<1$ 
holds for all $h$ in the interval $\left(0, h^{*}\right]$. Let $h \in\left(0, h^{*}\right]$.

3. Now conclude from $(32)$ and $\rho \in(0,1)$ that

$$
\frac{3 \rho^{2}}{\nu^{2}\left(1-\frac{2}{3} \rho+\frac{2}{3} \rho^{2}\right)}\left|w^{0}\right|_{H}^{2} \leq \frac{\rho}{6}(1-\rho)<\frac{1}{4}
$$

which together with (c) implies that

$$
\hat{c}_{2}(\rho, h, z)<1 \text { and } \frac{\hat{c}_{2}(\rho, h, z)}{c_{1}(\rho, h, z)} \leq \kappa_{2}<1 .
$$

Furthermore, (a) and (b) give $c_{2}^{0}(\rho, h, z) \leq 1$, so that with (31)

$$
\left|w^{1}\right|_{V}^{2} \leq \frac{2 c_{2}^{0}(\rho, h, z)}{\nu h\left(1-\frac{2}{3} \rho+\frac{2}{3} \rho^{2}\right)}\left|w^{0}\right|_{H}^{2} \leq \frac{2}{\nu h\left(1-\frac{2}{3} \rho+\frac{2}{3} \rho^{2}\right)} \kappa^{0}\left|w^{0}\right|^{2} .
$$

4. Now assume that for $j \in \mathbb{N}$

(a) $\left|w^{j}\right|_{H}^{2} \leq \kappa^{j}\left|w^{0}\right|_{H}^{2}$ and

(b) $\left|w^{j}\right|_{V}^{2} \leq \frac{2}{\nu h\left(1-\frac{2}{3} \rho+\frac{2}{3} \rho^{2}\right)} \kappa^{j-1}\left|w^{0}\right|_{H}^{2}$

holds true, where $\kappa:=\max \left(\kappa_{1}, \kappa_{2}\right)<1$.

5. Then conclude from (b)

$$
\begin{aligned}
c_{2}^{j}(\rho, h, z) & =\frac{1}{2}+\frac{3 h \rho^{2}}{2 \nu}\left|w^{j}\right|_{V}^{2}+\tilde{c}_{2}(\rho, h, z) \\
& \leq \frac{1}{2}+\frac{3 h \rho^{2}}{2 \nu} \frac{2}{\nu h\left(1-\frac{2}{3} \rho+\frac{2}{3} \rho^{2}\right)} \kappa^{j-1}\left|w^{0}\right|_{H}^{2}+\tilde{c}_{2}(\rho, h, z) \\
& \leq \hat{c}_{2}(\rho, h, z) .
\end{aligned}
$$

Thus, utilizing (31) one more time gives

$$
\left|w^{j+1}\right|_{H}^{2} \leq \frac{c_{2}^{j}(\rho, h, z)}{c_{1}(\rho, h, z)}\left|w^{j}\right|_{H}^{2} \leq \frac{\hat{c}_{2}(\rho, h, z)}{c_{1}(\rho, h, z)}\left|w^{j}\right|_{H}^{2} \leq \kappa^{j+1}\left|w^{0}\right|_{H}^{2}
$$

and

$$
\left|w^{j+1}\right|_{V}^{2} \leq \frac{2}{\nu h\left(1-\frac{2}{3} \rho+\frac{2}{3} \rho^{2}\right)} c_{2}^{j}(\rho, h, z)\left|w^{j}\right|_{H}^{2} \leq \frac{2}{\nu h\left(1-\frac{2}{3} \rho+\frac{2}{3} \rho^{2}\right)} \kappa^{j}\left|w^{0}\right|_{H}^{2},
$$

which completes the proof of Theorem 8 .

Remark 3. 1. In the discrete scheme (29) all local quantities are discretized implicitly, non-local quantities explicitely.

2. The proof of Theorem 8 is constructive. Estimates of $\rho^{*}$ and $h^{*}(\rho)$ therefore can be deduced from (32) and 2. (a)-(d), respectively.

3. Note further, that the conditions on $\rho$ in (21), (24) are satisfied for the stepsize of (13), provided the parameter $\alpha$ is chosen sufficiently large. 


\section{Numerical validation}

Here the results obtained in the previous sections are numerically validated. In order to value the performance of the feedback operators (19) and (20) the numerical example is taken from [16]. As is demonstrated below the instantaneous controller presented here steers the H-norm and the $\mathrm{V}$-norm of the difference $y-z$ to zero with exponential decay. This seems to be a more stable performance than that reported by Hou and Yan in [16] for their (1,1)-receding horizon controller (i.e. control horizon length coincides with time step size). The instantaneous controls are compared to the optimal control and it turns out that instantaneous controls give a much better reduction of the control gain, but at significant higher overall costs.

The control problem considered here is of tracking type and is given by $(\mathbf{P})$ with cost functional

$$
J(y, u):=\frac{1}{2} \int_{Q}|y-z|^{2} d x d t+\frac{\alpha}{2} \int_{Q}|u|^{2} d x d t
$$

and control space $\mathcal{U}:=L^{2}(\Omega)^{2}$, with $\mathcal{B}$ denoting the injection from $U$ into $V^{\prime}$. The initial value of the uncontrolled flow is chosen as

$$
y(x, 0)=e\left[\begin{array}{c}
\left(\cos 2 \pi x_{1}-1\right) \sin 2 \pi x_{2} \\
-\left(\cos 2 \pi x_{2}-1\right) \sin 2 \pi x_{1}
\end{array}\right]
$$

with $e$ denoting the Euler number, and the desired state is time dependent and given by

$$
z(t, x)=\left[\begin{array}{c}
\varphi_{x_{2}}\left(t, x_{1}, x_{2}\right) \\
-\varphi_{x_{1}}\left(t, x_{1}, x_{2}\right)
\end{array}\right]
$$

where $\varphi$ is defined through the stream function

$$
\varphi\left(t, x_{1}, x_{2}\right)=\theta\left(t, x_{1}\right) \theta\left(t, x_{2}\right)
$$

with

$$
\theta(t, y)=(1-y)^{2}(1-\cos 2 k \pi t), \quad y \in[0,1]
$$

For the results presented $\alpha=1 . e-2, k=1$ and the time interval is chosen as [0,2], i.e. $T=2$. For the discretization in time an equidistant grid with width $\delta t=0.01$ is used, for the spatial discretization the Taylor-Hood finite element [15] is used on a grid containing 1024 triangles with 2113 velocity and 545 pressure nodes. The number of unknowns in the discretized control problem therefore has the magnitude $1.65 \times 10^{6}$, including the primal, adjoint and control variables.

In Fig. 1 the desired flow at $T=2$ is shown. It forms four cells with opposite flow directions near the cell boarders.

In Fig. 2 the evolution of the optimally controlled flow computed with Newton's method (see $[11,13]$ for computational details) and the instantaneously controlled flow is illustrated at selected time instances. The costs are compared in Fig. 3. For the instantaneous control strategy they become larger with increasing time. This is due to the increasing dynamics of the desired state. As is expected the optimal control strategy equi-distributes the costs over the time horizon, whereas instantaneous control at every time instance intends to match the desired state. This is also illustrated by the evolution in Fig. 2 .

For $\nu=1 / \operatorname{Re}=1 / 10$ and $\gamma=1 . e-2$ the numerical computation of the optimal control takes about 45 minutes cpu-time on a DEC-ALPHA ${ }^{T M}$ station 500. The instantaneous feedback controller takes about 2 minutes to compute a control function on the time horizon $[0,2]$.

In Fig. 4 the evolution of the $L^{2}$-cost for the instantaneous control law is shown for $\rho=0.1$ and different values of $h$. In Fig. $5 h=0.1$ is fix and the evolution of the control gain in the $L^{2}-$ and the $H^{1}$-norm for different values of $\rho$ are shown. Exponential decay is observed and thus, the theoretical results of Theorems 4, 5, 6 and 7 are confirmed. 


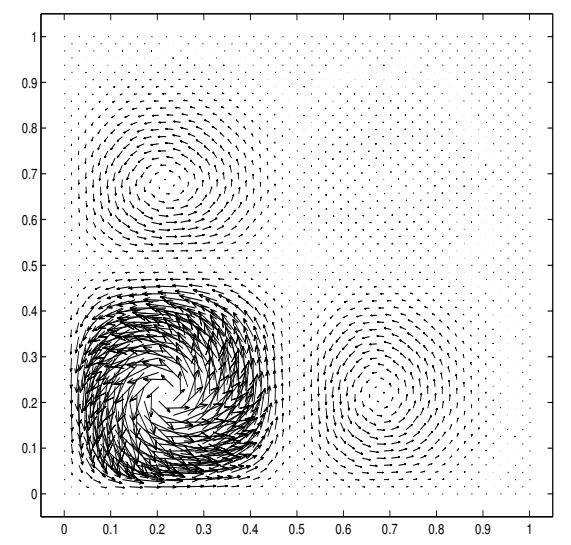

Figure 1: Desired flow at $T=2$

\section{Acknowledgment}

The author greatfully acknowledges the support of the Collaborative Research Center 609 (SFB 609) Elekrtomagnetische Strömungsbeeinflussung in Metallurgie, Kristallzüchtung und Elektrochemie funded by the German Research Foundation (DFG).

\section{References}

[1] Abergel, F. \& Temam, R. On some Control Problems in Fluid Mechanics. Theoret. Comput. Fluid Dynamics, 1:303-325, 1990.

[2] Berggren, M. Numerical solution of a flow control problem: Vorticity reduction by dynamic boundary action. Siam J. Sci. Comput., Vol. 19(No. 3):829-860, 1998.

[3] Choi, H. Suboptimal Control of Turbulent Flow Using Control Theory. In Proceedings of the International Symposium. on Mathematical Modelling of Turbulent Flows, 1995. Tokyo, Japan.

[4] Choi, H.; Hinze, M. \& Kunisch, K. Instantaneous control of backward-facing-step flows. Applied Numerical Mathematics, 31:133-158, 1999.

[5] Choi, H; Temam, R.; Moin, P. \& Kim, J. Feedback control for unsteady flow and its application to the stochastic Burgers equation. J. Fluid Mech., 253:509-543, 1993.

[6] Constantin, P. \& Foias, C. Navier-Stokes Equations. The University of Chicago Press, 1988.

[7] García, C.E.; Prett, D.M. \& Morari, M. Model predictive control: Theory and practice - a survey. Automatica, 25(3):335-348, 1989.

[8] Gunzburger, M.D. \& Manservisi, S. Analysis and approximation for linear feedback control for tracking the velocity in Navier-Stokes flows. Comput. Methods Appl. Mech. Eng., 189:803-823, 2000. 



Figure 2: Optimally controlled (left) versus instantaneously controlled flows for $t=0.1,1 ., 1.6,2$. 

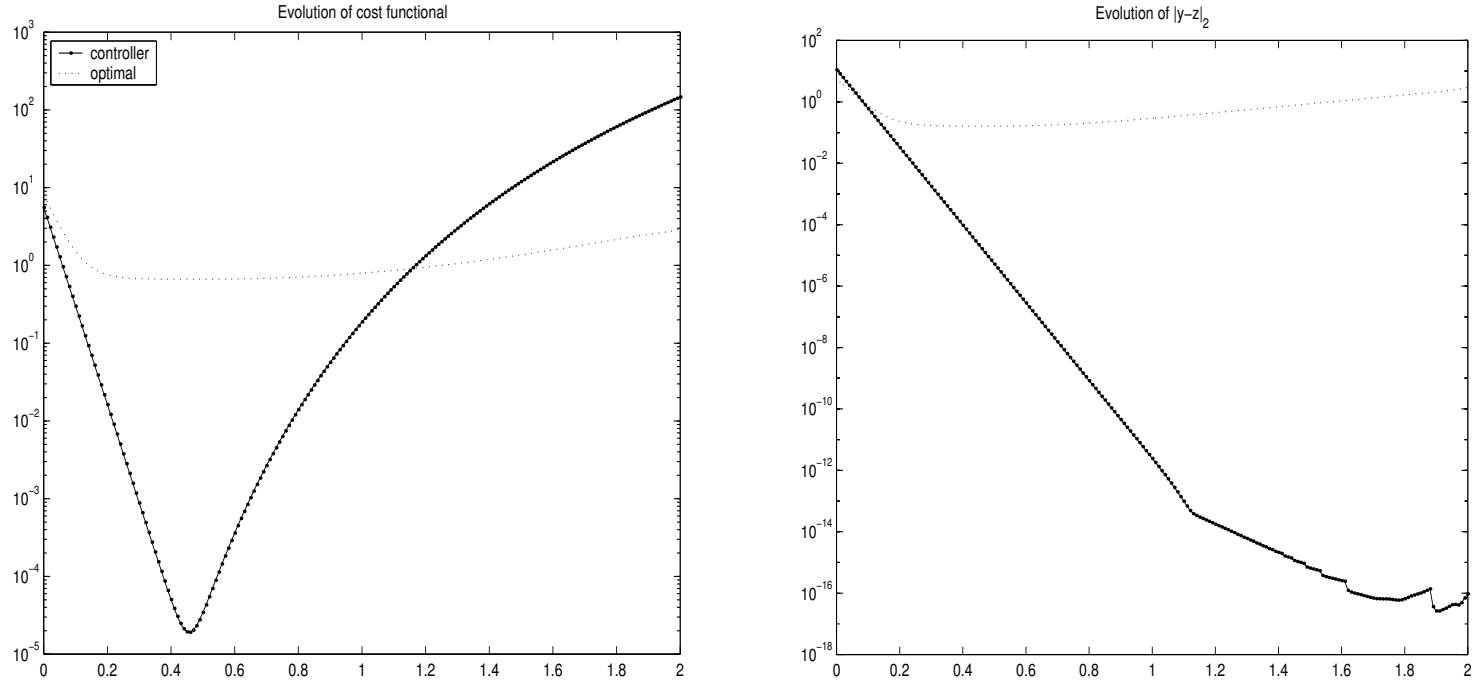

Figure 3: Evolution of cost (left) and control gain for $h=0.01$ and $\rho=0.1$
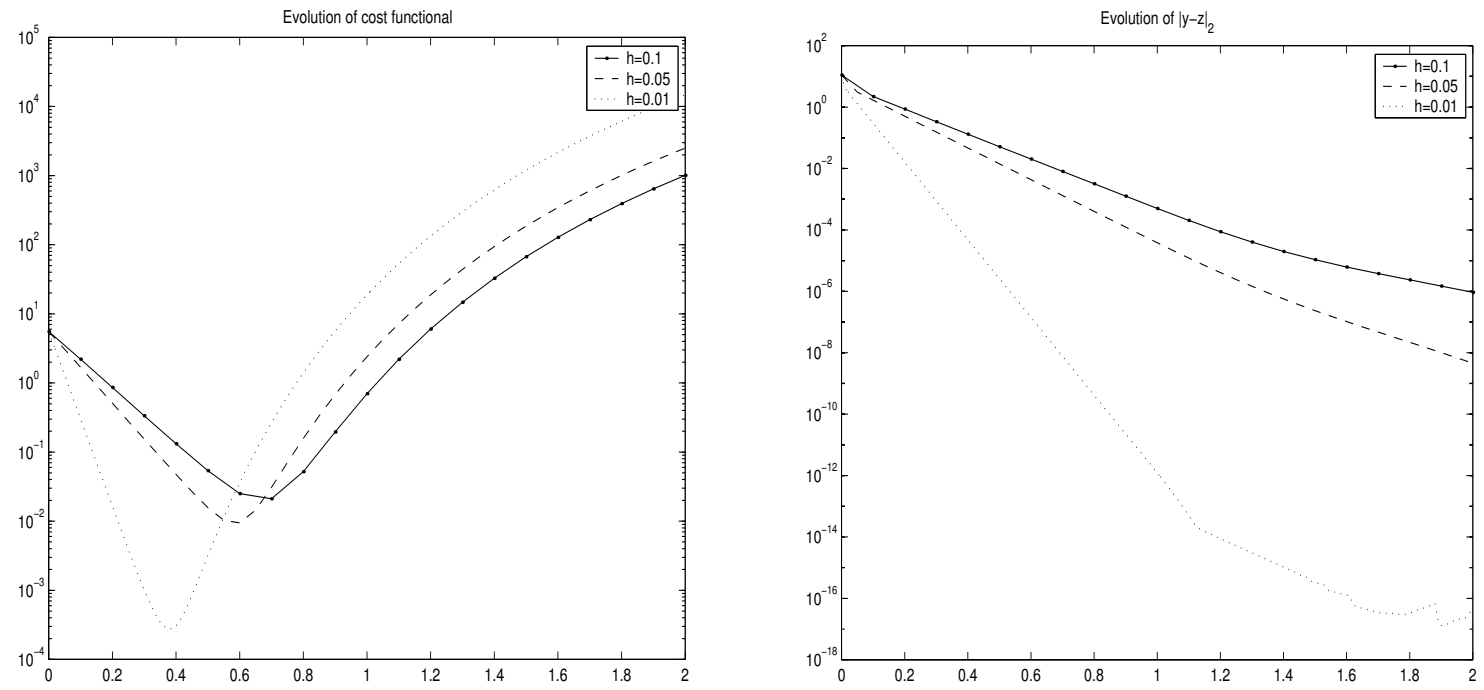

Figure 4: Evolution of cost (left) and control gain for $\rho=0.1$ and $h=0.01,0.05,0.1$ 

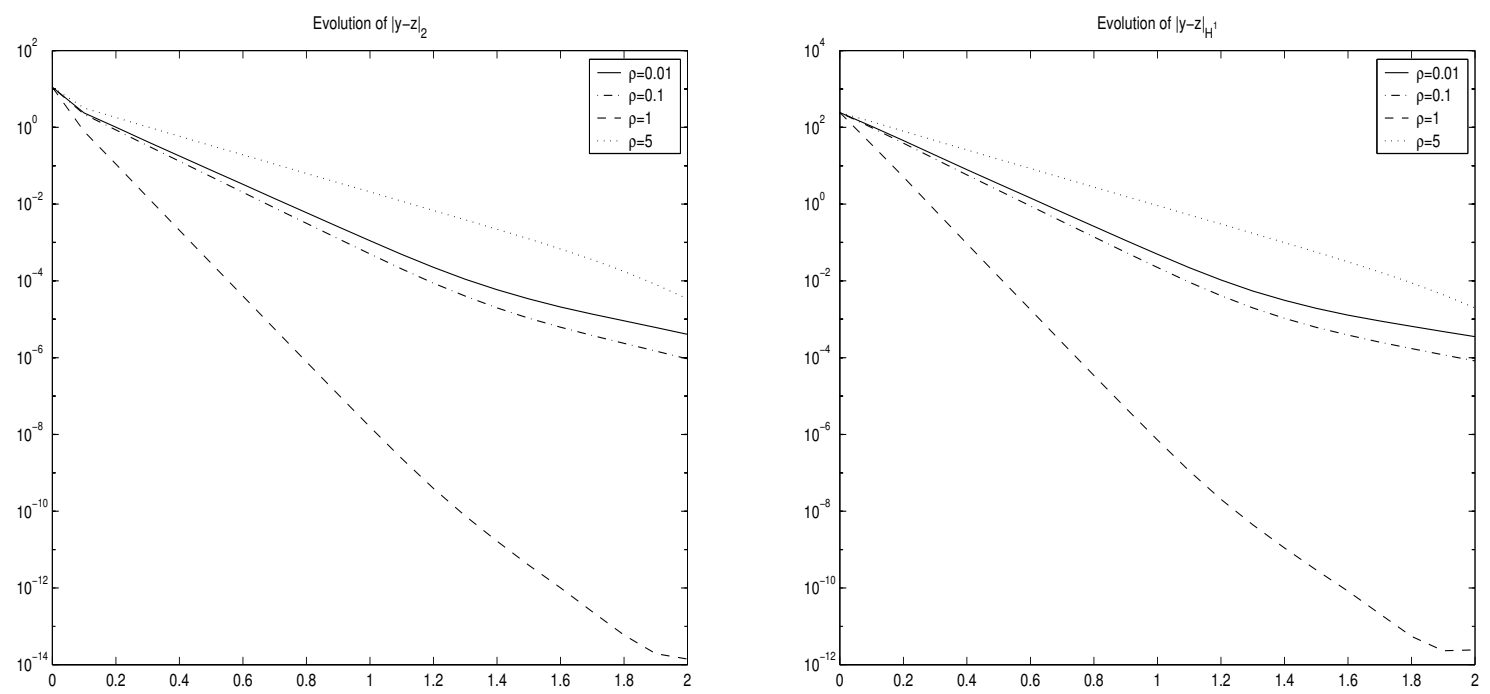

Figure 5: Evolution of control gain in $L^{2}$-norm (left) and $H^{1}$-norm for $h=0.1$ and $\rho=$ $0.01,0.1,1 ., 5$.

[9] Gunzburger, M.D. \& Manservisi, S. Analysis and approximation of the velocity tracking problem for Navier-Stokes flows with distributed control. Siam J. Numer. Anal., 37:1481-1512, 2000.

[10] Hill, D.C. Drag reduction strategies. CTR Annual Research Briefs, 1993. Center for Turbulence Research, Stanford University/NASA Ames Research Center, 3-14.

[11] Hinze, M. Optimal and instantaneous control of the instationary Navier-Stokes equations. Habilitationsschrift, 1999. Fachbereich Mathematik, Technische Universität Berlin, revised version see http://www.math.tu-dresden.de/ ${ }^{\sim}$ hinze/publications.html.

[12] Hinze, M. \& Kunisch, K. Control strategies for fluid flows - optimal versus suboptimal control. In H.G.Bock et al., editor, ENUMATH 97, pages 351-358. World Scientific, 1997. Singapore.

[13] Hinze, M. \& Kunisch, K. Second order methods for optimal control of time-dependent fluid flow. SIAM J. Control Optim., 40:925-946, 2001.

[14] Hinze, M. \& Volkwein, S. Instantaneous control for the Burgers equation: Convergence anlysis and numerical implementation. Nonlinear Analysis T.M.A., 50:1-26, 2002.

[15] Hood, P. \& Taylor, C. A numerical soution of the Navier-Stokes equations using the finite element technique. Comp. and Fluids, 1:73-100, 1973.

[16] Hou, L.S. \& Yan, Y. Dynamics and approximations of a velocity tracking problem for the Navier-Stokes flows with piecewise distributed controls. SIAM J. Control Optim., 35:1847$1185,1997$.

[17] Hou, L.S. \& Yan, Y. Dynamics for controlled Navier-Stokes Systems with distributed controls. SIAM J. Control Optim., 35:654-677, 1997. 
[18] Kauffmann, A. \& Kunisch, K. Optimal control of a solid fuel ignition model. ESAIM:Proceedings, 8:65-76, 2000.

[19] Lee, C.; Kim, J. \& Choi, H. Suboptimal control of turbulent channel flow for drag reduction. J. Fluid Mech., 358:245-258, 1998.

[20] Min, C. \& Choi, H. Suboptimal feedback control of vortex shedding at low Reynolds numbers. J. Fluid Mech., 401:123 - 156, 1999.

[21] Nevistić, V. \& Primbs, J.A. Finite receding horizon control: A general framework for stability and performance analysis. Preprint, 1997. Automatic Control Laboratory, ETH Zürich, Switzerland.

[22] Rawlings, J.B. \& Muske, K.R. The stability of constrained receding horizon control. IEEE Transactions on Automatic Control, 38(10):1512-1516, 1993.

[23] Temam, R. Navier-Stokes Equations. North-Holland, 1979.

[24] Temam, R. Infinite-Dimensional Dynamical Systems in Mechanics and Physics, Second Edition. Springer, 1997.

[25] Tröltzsch, F. \& Unger, A. Fast solution of optimal control problems in selective cooling of steel. ZAMM, 81:447-456, 2001. 